\title{
Angiotensin II Silencing Alleviates Erectile Dysfunction Through Down-Regulating the Rhoa/Rho Kinase Signaling Pathway in Rats with Diabetes Mellitus
}

\author{
Ying Zhang ${ }^{a}$ Linpei Jiab Ying Zhang ${ }^{c}$ Wei Jid Hai Lie \\ aDepartment of Pathology, China-Japan Union Hospital of Jilin University, Changchun, ${ }^{b}$ Department \\ of Nephrology, Xuanwu Hospital, Capital Medical University, Beijing, 'Department of Neurology, First \\ Hospital of Jilin University, Changchun, 'Department of Vascular Surgery, Jilin Provincial People's \\ Hospital, Changchun, e'Department of Urology, China-Japan Union Hospital of Jilin University, \\ Changchun, China
}

\section{Key Words}

Angii • RhoA/Rho kinase signaling pathway • Diabetes mellitus • Erectile dysfunction

\begin{abstract}
Background/Aims: We aim to explore the role of angiotensin (Ang)II and the RhoA/Rho kinase signaling pathway in the pathogenesis of erectile dysfunction in diabetes mellitus (DM). Methods: Male Sprague-Dawley (SD) rats were used for experiments and short hairpin RNA (shRNA) was used to silence the AngII gene. The erectile function of rats was observed and intracavernous pressure and mean arterial pressure (ICP/MAP) were measured after electrical stimulation. Relaxation and contraction of smooth muscle in the corpus cavernosum were tested. Western blotting and quantitative RT-PCR were applied to measure the expressions of RhoA, Rho-associated kinase (ROCK)1 and ROCK2. Radioimmunoassay was applied to detect the levels of AngII. Results: Rats in the control group had the most erectile times, followed by AngII-silenced rats with DMED and rats with DMED. Rats with DMED had worse ICP and MAP than AngII-silenced rats. The contraction ability was markedly improved and relaxation ability was decreased in AngII-silenced rats with DMED as compared with rats with DMED. The levels of AngII were significantly increased in DMED rats while significantly decreased after AngII silencing. The mRNA and proteins of RhoA and ROCK2 were expressed in a similar way. Conclusion: AngII silencing improves erectile dysfunction via down-regulating the RhoA/Rho kinase signaling pathway.
\end{abstract}




\section{Introduction}

Diabetes mellitus (DM) refers to a series of metabolic disorders which mainly includes type $1 \mathrm{DM}$ due to dysfunction of beta cell of pancreas and type $2 \mathrm{DM}$ caused by insulin resistance and relative insulin deficiency [1]. According to the data from International Diabetes Federation, DM affects more than 285 million people globally, and this number is expected to increase to 438 million in 2030 [2]. The long-term complications of DM include heart disease, stroke, nephropathy, foot ulcers, and damage to the eyes, etc [3-5]. Among others, erectile dysfunction (ED) has long been recognized as a common complication of DM [3-5]. ED or impotence is a kind of sexual dysfunction characterized by the inability to develop or maintain the erection of penis during sexual activity [6]. Organic ED may result from DM and hypertension, as well as unhealthy common lifestyle factors, such as obesity, lack of physical exercise, and lower urinary tract symptoms [6]. Serial actions of vascular endothelial cells, smooth muscle cells, pericytes, and autonomic nerves are implicated in penile erection [7]. A recent study has revealed that the inhibition of the A2B adenosine receptor signaling pathway resulted in ED in rats with DM [8]. In this regard, the effects of signaling pathways with cardiovascular relevance are worth investigating in DM-induced ED.

Angiotensin (Ang) is a kind of peptide hormone which can cause vasocontraction and a subsequent elevation in blood pressure level. Among others, the majority actions of angiotensin II (AngII) were stimulated by AngII type 1 receptor, one of the major receptors binding to AngII [9]. In addition to the role of AngII in inflammation, tissue injury, autoimmunity, oxidative stress [10], AngII receptors were expressed throughout the nephron, and their activation was reported to associate with salt and water reabsorption, which is crucial in the maintenance of blood pressure [11]. Moreover, abnormal expressions of AngII were associated with changes in cardiovascular function [12]. A previous study revealed an abnormal expression of RhoA/Rho-associated kinase (ROCK) signaling pathway in penile corpus cavernosum smooth muscles of rats with hyperlipidemia-induced ED [13]. Collectively, in light of the functions of AngII and the RhoA/ROCK signaling pathway in ED, we hypothesize that AngII and the RhoA/ROCK signaling pathway may act in cooperation in the pathogenesis of ED. Therefore, we investigated the effect of AngII and the RhoA/ROCK signaling pathway by in vivo silencing AngII in diabetic rats.

\section{Materials and Methods}

\section{Ethics statement}

Animal experiments were conducted in strict accordance with the approved animal protocols and guidelines established by the Ethics Committee of the China-Japan Union Hospital of Jilin University. All efforts were made to minimize the number and sufferings of experimental animals.

\section{Establishment of the DMED model}

A total of 50 clean male Sprague-Dawley (SD) rats with body weight ranging from $200 \mathrm{~g}$ to $250 \mathrm{~g}$ and with normal erectile function were housed on a 12-h light/dark cycle with standard chow and water ad libitum. Forty rats were randomly divided into the model group for the establishment of the DMED model (DM rats with erectile dysfunction). After fasted for $12 \mathrm{~h}$, rats were intraperitoneally injected with streptozotocin (STZ, $60 \mathrm{mg} / \mathrm{kg}$, Sigma, United States) in citrate buffer solution (0.1 mol/L, pH4.5) [8]. Ten rats (as controls) were intraperitoneally injected with the same volume of citrate buffer solution. Rats with non-fasting blood glucose level $>16.67 \mathrm{mmol} / \mathrm{L}$ were considered type 1 diabetic [14]. Eight weeks later, four rats died and the rest rats were used for further experiments. Apomorphine (APO, Sigma, United States) at a dosage of 100 $\mu \mathrm{g} / \mathrm{kg}$ body weight were injected into the rats and the penile erection within $0.5 \mathrm{~h}$ was recorded. Finally, a total of 30 rats developed DMEM were randomly divided into the DMED group, the DMED empty vector group (Ad-null group) and the DMED Ad-Ang-2 shRNA group. Specifically, in the DMED Ad-Ang-2 shRNA group, post-transcriptional translation of AngII was blocked, which is mediated by small hairpin (sh)RNAs.

\section{KARGER}




\section{Cellular Physiology Cell Physiol Biochem 2018;45:419-427 \begin{tabular}{ll|l} 
and Biochemistry Published online: January 26, 2018 & $\begin{array}{l}\text { (c) } 2018 \text { The Author(s). Published by S. Karger AG, Basel } \\
\text { www.karger.com/cpb }\end{array}$
\end{tabular} \\ Zhang et al.: AngII Silencing in DMED Rats}

\section{Vector construction and medical treatment}

Six AngII shRNAs were designed according to the sequences of Genebank database and protocols from previous studies in order to select the two optimal sequences [15]. In brief, the expression vector was purchased from System Biosciences (Mountain View, CA, USA). The sequence was synthesized by Shanghai Sangon Biological Engineering Technology and Services Co., Ltd. (Shanghai, China). After sequence synthesis, the viral vector was digested and linked. The connected product will be transfected into E.coli TOP10 chemically competent cells, followed by PCR identification, positive clone sequencing and plasmid extraction. HEK293 cells were used to obtain recombinant adenovirus for the transfection of plasmid. The recombinant adenovirus Ad-Ang-2 shRNA (titer, $5.3 \times 10^{9} \mathrm{puf} / \mathrm{ml}$ ) and negative control adenovirus Ad-null (titer, $6.2 \times 10^{9} \mathrm{puf} / \mathrm{ml}$ ) (Wuhan Genesil Biotechnology Co., Ltd.) were constructed. The rats in the DMED group, Ad-null group and DMED Ad-Ang-2 shRNA group, in which Ang II was silenced, were intraperitoneally injected with pentobarbital sodium (35 mg/kg, Sigma, United States) for anesthesia. The surface skin in the penis was conventionally disinfected with iodophor and then the root of the penis was bound with a rubber band to stop the blood flow. After pilot studies, rats in the DMED Ad-Ang-2 shRNA group, Ad-null group and DMED group was injected with Ang-2 shRNA $(20 \mu \mathrm{L})$, Ad-null $(20 \mu \mathrm{L})$ and normal saline $(20 \mu \mathrm{L})$, respectively, to the corpus cavernosum. Meanwhile, both sides of the penis of rats in the DMED Ad-Ang-2 shRNA group, the Ad-null group and the DMED group were injected with $100 \mu \mathrm{L}$ of Ang-2 shRNA, Ad-null and normal saline, respectively. A message was conducted in the penis to promote the drug diffusion and $30 \mathrm{~min}$ later, the rubber band was released to recover the blood flow. The medication was injected in rats of each group every other day and intervention remained for 2 weeks.

\section{Observation of erectile function in rat models}

After two weeks, rats were injected with APO $(100 \mu \mathrm{g} / \mathrm{kg})$ into the neck skin. Five minutes later, the rats were under observation for half an hour with their glans exposed, prepuce backward and the penis erected. The erectile time was recorded. During the observation, obvious and non-random mouth open, as well as respiratory movement (yawn) were observed.

\section{Measurement of intracavernosal pressure (ICP) and mean arterial blood pressure (MAP)}

The neck skin of the rats was separated to insert the carotid artery intubation. A median incision was made in the venter inferior to expose the ganglion of the pelvic cavity and the nerve of the corpus cavernosum which was stimulated by the double claw electrodes $(5.0 \mathrm{mV}$; frequency, $20 \mathrm{~Hz}$; amplitude, 5 $\mathrm{ms}$, time, $1 \mathrm{~min}$ ). The $23 \mathrm{G}$ probe containing $250 \mathrm{IU} / \mathrm{ml}$ of heparin was inserted into the crura penis. The intubation and probe were connected with pressure transmitters to detect the MAP and the ICP values.

\section{In vitro relaxation and contraction of the smooth muscle in corpus cavernosum}

Rats were anesthetized with their penis removed. Part of the tissues were used for further experiments and the rest tissues were soaked in Krebs medium at $4{ }^{\circ} \mathrm{C}$ to separate the cavernous body of urethra, cartilage penis, nerve and blood vessels. Later, the corpus cavernosum was exposed for preparation of $6 \mathrm{~mm} \times 2 \mathrm{~mm}$ $\times 2 \mathrm{~mm}$ strips, the ends of which were fixed in thermostatic bath and tonotransducer respectively. The tension $\mathrm{F}_{2}$ was measured once the strip was balanced and the tension $\mathrm{F}_{1}$ was measured after $50 \mu \mathrm{mol} / \mathrm{L}$ of phenylephrine $(\mathrm{PE})$ was added. The contraction percentage was calculated as $\left(\mathrm{F}_{1}-\mathrm{F}_{2}\right) / \mathrm{F}_{1}$. The strips were then rinsed in Krebs medium to eliminate the PE and $100 \mu \mathrm{mol} / \mathrm{L}$ of Ach was added to measure tension $\mathrm{F}_{3}$. The relaxation percentage was calculated as $\left(\mathrm{F}_{1}-\mathrm{F}_{3}\right) / \mathrm{F}_{1}$.

\section{Western blotting}

Total protein was extracted and the protein concentration was detected by using the bicinchoninic acid (BCA) method. $40 \mu \mathrm{g}$ of protein was collected, separated by 10\% SDS-PAGE, and then transferred to the polyvinylidene fluoride (PVDF) membrane. Subsequently, 5\% of skim milk power was added to the membrane at $37^{\circ} \mathrm{C}$ and was sealed at room temperature for $1 \mathrm{~h}$. Rabbit anti-primary antibodies of Rho A (Abcam, United States), ROCK1 (Abcam, United States), ROCK2 (Santa Cruz, United States) and $\beta$-actin (Santa Cruz, United States) were diluted to $1: 300$ and added to the membrane for incubation at $4^{\circ} \mathrm{C}$ overnight. TBST was used to wash the membrane and the HRP-labeled goat anti-rabbit and goat anti-mouse IgG antibodies (diluted 1:5000) were added and incubated at $37^{\circ} \mathrm{C}$ for $1 \mathrm{~h}$. After that, the membrane was rinsed for 3 times (10 min each time). The secondary antibodies of ${ }^{125}$ I labeled goat anti-rabbit and goat anti-mouse (Wuhan 
Zhang et al.: AngII Silencing in DMED Rats

Table 1. The primer sequences for reverse transcription quantitative polymerase chain reaction

\begin{tabular}{|c|c|c|}
\hline Gene & Upstream & Downstream \\
\hline Rho A & 5'-GCAGGTAGAGTTGGCTTTATGG-3' & 5'-CTTGTGTGCTCATCATTCCGA-3' \\
\hline ROCK1 & 5'-GAATGACATGCAAGCGCAAT-3' & 5'-GTC CAAAAGTTTTGCACGCA-3 \\
\hline ROCK2 & 5'-GAAACAACTGGATGAAGCTAATGC-3' & 5'-GTTTCAAGCAGGCAGTTTTTATCTT-3' \\
\hline GAPDH & 5'-CCATGCCATCACTGCCACT-3' & 5'-TGTCATCATACTTGGCAGGTTTC-3' \\
\hline
\end{tabular}

Kerui Science and Technology Ltd., Hubei, China) were added according to the instruction for incubation for $1 \mathrm{~h}$. Then the membrane was washed for 3 times (10 min each time) and developed by using ECL. The results were analyzed by using the Quantity One software.

\section{Determination of AngII in the blood of corpus cavernosum}

The concentrations of AngII in the blood of corpus cavernosum were determined using an AngII radioimmunoassay kit from the North Biotech Company in China. By adopting the competition mechanism, the AngII in the standard medium and ${ }^{125}$ I-AngII added with radioimmunoassay reagent were competitive to react with the certain quantitated specific antibody. The immune separation reagent was used to separate the separation part (F) and the binding part (B) to further measure the radiation intensity of the binding part and calculate the binding rate $\mathrm{B} / \mathrm{B}_{0}$. The standard inhibitory curve was obtained after calculation with standard AngII content and corresponding binding rate. The AngII level in the sample was determined by checking the standard inhibitory curve.

\section{Real-time polymerase chain reaction}

The smooth muscle tissues in cavernous from each group were collected and grinded with liquid nitrogen. RNAiso Plus was added for dissociation. Total RNA were extracted according to the introduction. The integrity of RNA was measured by RNA electrophoresis. RNA density and purity were calculated based on the OD 260/280 value of RNA samples detected using an ultraviolet spectroscopy. Reverse transcription kit (Takara, Kyoto, Japan) was used for reverse transcription into cDNA. Using cDNA as template, SYBR GREEN fluorescent reagent was used for real-time PCR (primer sequences as shown in Table 1). The total volume of PCR was $10 \mu \mathrm{L}$. The amplification was conducted under following conditions: total 40 cycles of $95^{\circ} \mathrm{C}$ for $30 \mathrm{~s}, 95^{\circ} \mathrm{C}$ for $5 \mathrm{~s}$ and $55^{\circ} \mathrm{C}$ for $30 \mathrm{~s}$. The attached melting curve of the software was used to observe the specificity of the amplified product. The expression of target protein was quantified by GAPDH expression. Each sample was set to three duplicated wells and the $2^{-\Delta \Delta c t}$ method was used to calculate the expressions among groups.

\section{Statistics}

The Statistical Program for Social Sciences (SPSS) 19.0 software (SPSS, IBM, West Grove, PA, USA) was used for data analysis. The measurement data was expressed as mean \pm standard deviation (mean $\pm \mathrm{SD}$ ). The one-way ANOVA was used to compare variables among multiple groups. The student- $t$ test was conducted for comparisons between two groups. $P<0.05$ was considered statistically significant.

\section{Results}

Establishment of DM rat models and selection of ED rats

Ten rats died in the process of modeling. Compared with rats in the control group, the DM rats drank and ate more and presented

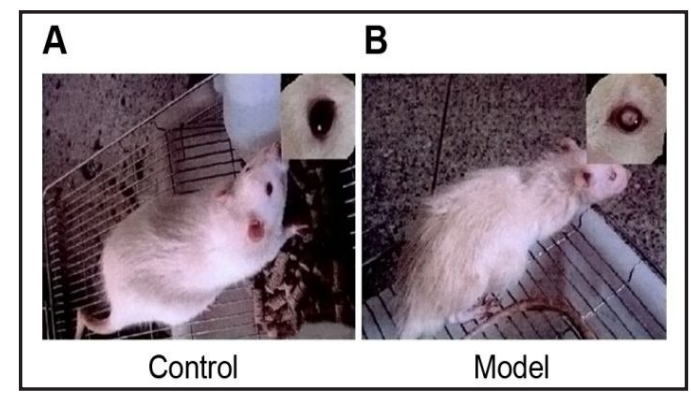

Fig. 1. Establishment of the DM model. Clean male Sprague-Dawley (SD) rats with body weight of 200-250 g were used for the establishment of DM model. After fasted for $12 \mathrm{~h}$, animals were intraperitoneally injected with streptozotocin $(60 \mathrm{mg} /$ kg body weight). Rats with non-fasting blood glucose level > $16.67 \mathrm{mmol} / \mathrm{L}$ were considered type 2 diabetic. The DM rats drank and ate more and presented with more urine and less shining fur with more dirt (B) as compared to normal rats (A). DM, diabetes mellitus. 
Table 2. Comparisons of blood glucose and weight in rats between the model and control groups. Note: ${ }^{*} \mathrm{P}$ $<0.05$ compared with the control group

\begin{tabular}{lccccc}
\hline \multirow{2}{*}{ Groups } & \multirow{2}{c}{ Blood glucose $(\mathrm{mmol} / \mathrm{l})$} & \multicolumn{2}{c}{ Weight $(\mathrm{g})$} \\
& & Before modeling & After modeling & Before modeling & After modeling \\
\hline Control group & 10 & $6.47 \pm 1.07$ & $5.86 \pm 1.03$ & $223.83 \pm 7.74$ & $452.65 \pm 22.31$ \\
Model group & 30 & $5.99 \pm 1.05$ & $22.31 \pm 3.32^{*}$ & $223.27 \pm 7.62$ & $266.75 \pm 32.04^{*}$ \\
\hline
\end{tabular}

with more urine and less shining fur with more dirt (Fig. 1). Four rats died of ketoacidosis and the DM model was successfully established in the left 30 rats (fasting blood glucose $>16.67$ $\mathrm{mmol} / \mathrm{L} 72 \mathrm{~h}$ later) with a successful rate of $75 \%$. The 30 diabetic rats were randomly Table 3. Comparisons of erection function. Note: $*, \mathrm{P}<0.05$ compared with the control group; \#, $\mathrm{P}<0.05$ compared with the DMED group and the Ad-null group

\begin{tabular}{lccc}
\hline Groups & Cases (n) & Erection time (min) & ICP/MAP (\%) \\
\hline Control group & 10 & $2.5 \pm 0.85$ & $73.45 \pm 11.15$ \\
DMED group & 10 & $0.3 \pm 0.48^{*}$ & $25.15 \pm 6.05^{*}$ \\
Ad-null group & 10 & $0.4 \pm 0.52^{*}$ & $22.59 \pm 5.7^{*}$ \\
DMED Ad-Ang-2 shRNA group & 10 & $1.3 \pm 0.67^{*} \#$ & $57.12 \pm 9.5^{*} \#$ \\
\hline
\end{tabular}

Table 4. Comparisons of contraction and relaxation. Note: ${ }^{*}, \mathrm{P}<0.05$ compared with the control group; \#, $\mathrm{P}<0.05$ compared with the DMED group

\begin{tabular}{lccc}
\hline Groups & Case (n) & Contraction (\%) & Relaxation (\%) \\
\hline Control group & 10 & $11.03 \pm 3.57$ & $40.84 \pm 10.38$ \\
DMED group & 10 & $23.75 \pm 6.64^{*}$ & $24.02 \pm 6.31^{*}$ \\
Ad-null group & 10 & $24.41 \pm 6.78^{*}$ & $24.81 \pm 6.63^{*}$ \\
DMED Ad-Ang-2 shRNA group & 10 & $15.00 \pm 5.21 \#$ & $35.69 \pm 7.84 \#$ \\
\hline
\end{tabular}

divided into the

DMED group ( $\mathrm{n}$

$=10)$, the Ad-null group $(\mathrm{n}=10)$ and the DMED Ad-Ang-2 shRNA group $(\mathrm{n}=10)$. As shown in Table 2, the blood glucose of DM rats was significantly higher than that in the control group, while the weight in DM rats was lower (both $P<0.05$ ). APO was used to determine the erectile function. Rats with no less than one erection were considered with erectile ability and were not included as the DMED model.

\section{AngII silencing promotes erectile function}

The erectile times of rats in the DMED group and the Ad-null group were less than the controls $(P<0.01)$. The erectile times of rats in the DMED Ad-Ang-2 shRNA group were less than the controls, while were more than those in the DMED group and the Ad-null group $(P<0.05)$. Comparisons between the DMED group and Ad-null group showed insignificant difference $(P>0.05)$. Compared with the controls, the erectile times of rats in the DMED group and the Ad-null group were decreased $(P<0.01)$, while the erectile function of rats in the DMED Ad-Ang-2 shRNA group was much improved when compared with that in the DMED group and Ad-null group $(P<0.05)(P<0.01)$ (Table 3$)$.

\section{AngII silencing suppresses contraction and increases relaxation function}

In comparisons with the control group and DMED Ad-Ang-2 shRNA group, the contraction function of rats in the DMED group and Ad-null group was obviously increased (all $P<0.05$ ). No significant difference between the control group and the DMED Ad-Ang-2 shRNA group was detected. In terms of the relaxation function, rats in the DMED group and the Ad-null group presented with decreased relaxation function when compared with those in the control group and the DMED Ad-Ang-2 shRNA group (all $P<0.05$ ). The relaxation function of rats between the control group and the DMED Ad-Ang-2 shRNA group was insignificant (Table 4). 


\section{Cellular Physiology

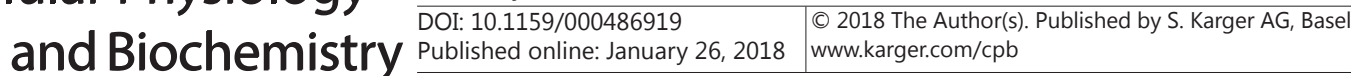 \\ Zhang et al.: AngII Silencing in DMED Rats}

Fig. 2. Concentrations of AngII in the corpus blood. The concentrations of AngII in the blood of the corpus cavernosum were determined by using radioimmunoassay. Among the four groups, rats in the DMED group and the Ad-null group had the highest levels of AngII, followed by the control group, and the DMED Ad-Ang-2 shRNA group had the lowest levels of AngII (all $\mathrm{P}<0.05$ ). *, $\mathrm{P}<0.05$ compared with the control group; \#, $\mathrm{P}<0.05$ compared with the Adnull group.
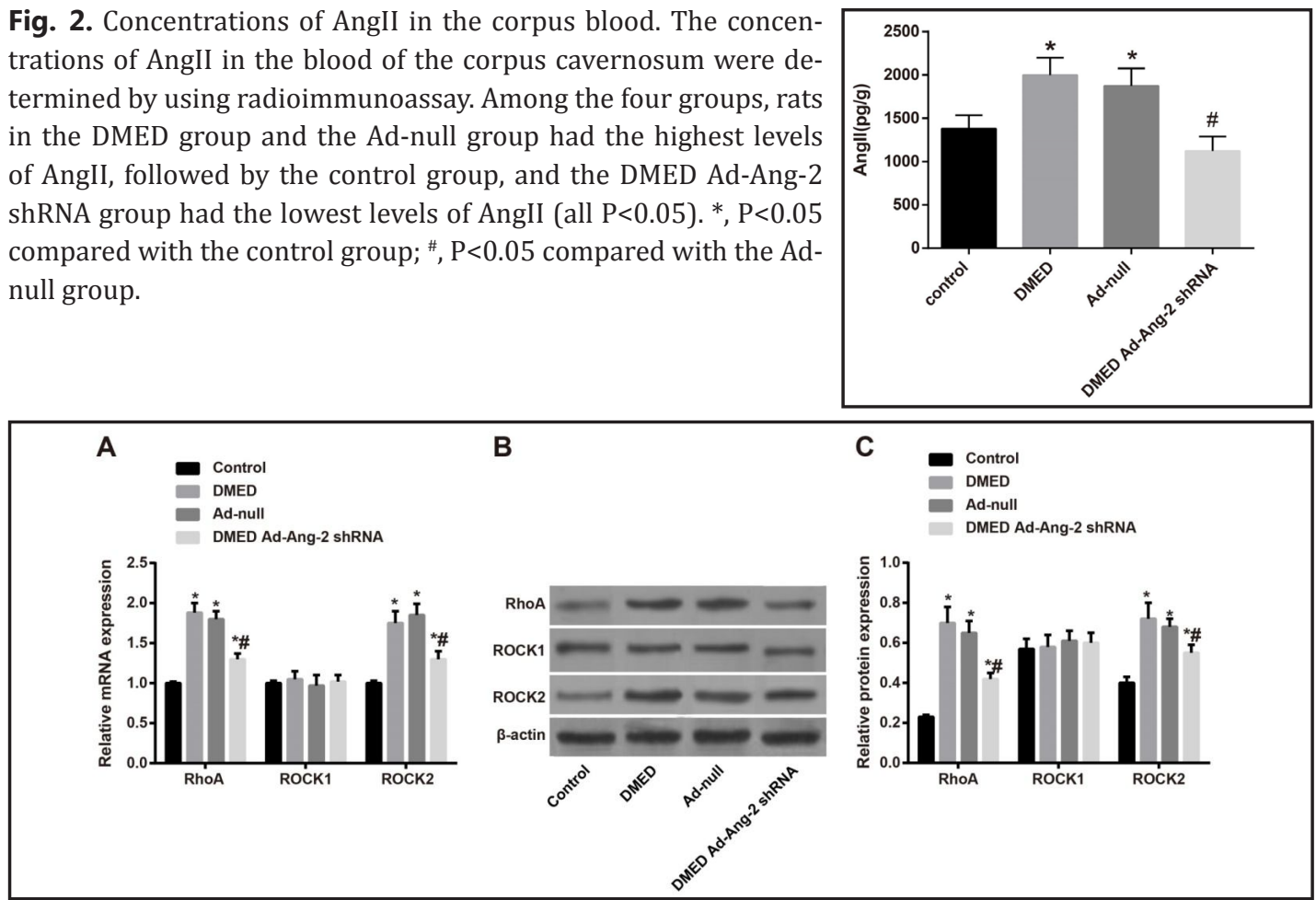

Fig. 3. Expressions of the RhoA/Rho kinase signaling pathway-related proteins. Western blotting and realtime polymerase chain reaction were applied to measure the expressions of RhoA, ROCK1 and ROCK2. A, the mRNA expressions of RhoA, ROCK1 and ROCK2; B, the protein expressions of RhoA, ROCK1 and ROCK2. The mRNA and protein expressions of RhoA and ROCK2 in the DMED group were significantly higher than those in the control group and in the DMED Ad-Ang-2 shRNA group (all $\mathrm{P}<0.05$ ). The mRNA and protein expressions of RhoA and ROCK2 in the DMED Ad-Ang-2 shRNA group were higher than that in the control group (both $\mathrm{P}<0.05$ ). The expressions of ROCK1 among the four groups showed no significant difference ( $\mathrm{P}$ $>0.05)$. DMED, diabetes mellitus induced erectile dysfunction; *, compared with the control group, $\mathrm{P}<0.05$;

\#, compared with the Ad-null group, $\mathrm{P}<0.05$.

\section{Concentrations of AngII in blood of the corpus cavernosum}

The concentrations of AngII in the blood of the corpus tissues among the four groups are presented in Fig. 2. Among the four groups, rats in the DMED group and the Ad-null group had the highest levels of AngII, followed by the control group, and the DMED Ad-Ang-2 shRNA group had the lowest levels of AngII (all $P<0.05$ ). Those results suggested that the Ang-2 shRNA vector was successfully expressed in rats and the expression of AngII in rats of the silencing group was remarkably suppressed than normal rats.

AngII silencing decreases the expressions of the RhoA/Rho kinase signaling pathwayrelated proteins

As shown in Fig. 3, the mRNA and protein expressions of RhoA and ROCK2 in the DMED group were significantly higher than those in the control group and in DMED Ad-Ang-2 shRNA group (all $P<0.05$ ), indicating that AngII silencing can reverse the up-regulation of the RhoA/Rho kinase signaling pathway caused by DM. The mRNA and protein expressions of RhoA and ROCK2 in the DMED Ad-Ang-2 shRNA group were higher than that in the control group (both $P<0.05$ ). The expressions of ROCK1 were insignificantly different among the four groups $(P>0.05)$, indicating that the abnormal regulation may exert its function on ROCK2 subtype. 


\section{Discussion}

This study addressed two key questions about DM-induced ED, namely, the effect of AngII silencing on DM-induced ED and the testing of the hypothesis that AngII and the RhoA/ROCK signaling pathway are involved in the pathogenesis of DM-induced ED. Accordingly, we used rat DMED models as an important tool for our experiment and our results showed that AngII silencing was successfully achieved via transfecting an Ad-Ang- 2 shRNA vector. Rats injected with Ad-Ang-2 shRNA vector had rather more erectile times and relaxation ability, as well as suppressed contraction ability when compared with rats in the DMED group and the Ad-null group. The expressions of RhoA and ROCK2 were inhibited in the Ad-Ang-2 shRNA group, but still higher than those in the control group.

The renin-angiotensin system (RAS) plays an important role in the maintenance of blood pressure and tissue perfusion [16]. RAS not only functions in the cardiovascular system, including regulation of blood pressure, but also in the central nervous system [9]. An estimate of $80 \%$ of male patients with chronic kidney disease were reported to have ED. Hypertensive patients often have elevated expressions of AngII and were reported with a higher risk for ED [17]. A previous study demonstrated that elevated AngII levels in the penile tissue were associated with the incidence of ED [17]. The possible explanation for the suppressed contraction ability in rats of the Ad-Ang-2 shRNA group may be that Ang-(1-7), a reactor of the ACE/AngII/AngII type 1 receptor, can offset the pressor actions mediated by AngII via the AT1 receptor [18-20]. These results grounded the speculation that the reactions among various AngII receptors could lead to the onset of abnormal cardiovascular functions, which may in turn trigger the occurrence of ED.

Meanwhile, the AngII type 2 receptor (AT2 receptor) does not conventionally correspond to reactors to elicit unusual signaling cascades which involve the activation of protein phosphatases and inhibition of protein kinases and RhoA GTPase [9]. RhoA is a GTP binding protein, which serves as a molecular switch between incompetence GDP and competence GDP [21]. The RhoA-GDP complex can bind to ROCK in its downstream to activate ROCK, so as to initiate the RhoA/ROCK pathway [22]. While the activated ROCK can phosphorylate the Myosin-Light-Chain Phosphatase (MLCP) to decrease the activity of MLCP, which could suppress the transformation into MLC, resulting in contraction in smooth muscle cells [23]. As long as the RhoA was transferred into non-active state, the activity of ROCK was correspondently decreased, which mediates the relaxation of smooth muscle cells [24]. Our results showed that the mRNA and protein expressions of RhoA and ROCK2 were lower in the Ad-Ang-2 shRNA group than those in the DMED group, but still higher those in the control group. Therefore, it is speculated that AngII silencing can down-regulate the RhoA/Rho kinase signaling pathway to improve the erectile function. Given the fact that insignificant difference on ROCK1 was detected among the control group, the Ad-Ang-2 shRNA group and the DMED group, AngII may regulate the RhoA/ROCK signaling pathway via the ROCK2 subtype, instead of ROCK1.

Although ED may result from factors like cardiovascular disease, prostatectomy, hormonal insufficiencies or drug side effects, our study only focused on the effect of AngII silencing mediated changes of the RhoA/Rho kinase signaling pathway in DMED. Interestingly, a recent study [25] showed that losartan, an AngII receptor antagonist improved erectile function in diabetic rats. Further studies are warranted both in animal models and in patients to determine the exact mechanism of known kinase signaling pathways and gene regulation relevant to ED [26].

In summary, our results supported the notion that the up-regulation of the RhoA/Rho kinase signaling pathway could decrease relaxation and increase contraction abilities of the cavernous smooth muscle, whereby contributing to the pathogenesis of ED in DMED rats. AngII silencing can down-regulate the RhoA/Rho kinase signaling pathway to improve the erectile function. Our silencing model may serve as a new approach for drug discovery and development of novel therapeutic modalities for ED.

\section{KARGER}




\title{
Cellular Physiology Cell Physiol Biochem 2018;45:419-427 \begin{tabular}{ll|l} 
DOI: 10.1159/000486919 26, 2018 & $\begin{array}{l}\text { O } 2018 \text { The Author(s). Published by S. Karger AG, Basel } \\
\text { www.karger.com/cpb }\end{array}$ \\
and Biochemistry Published online: January 26,
\end{tabular}
}

Zhang et al.: AngII Silencing in DMED Rats

\section{Acknowledgements}

The work was supported by grants from Jilin University.

\section{Disclosure Statement}

\author{
No Disclosure Statements exists.
}

\section{References}

1 Lee SH, Yu J: Clinical features of childhood diabetes mellitus focusing on latent autoimmune diabetes. Ann Pediatr Endocrinol Metab 2016;21:212-218.

2 Hu FB: Globalization of diabetes: the role of diet, lifestyle, and genes. Diabetes Care 2011;34:1249-1257.

-3 Elisaf M, Tzavela E, Karanatsis N, Tsimihodimos V: Antidiabetic Drugs and the Kidney. Curr Pharm Des 201 7;10.2174/1381612823666170307103222

-4 Li X, Lee YJ, Kim HY, Tan R, Park MC, Kang DG, Lee HS: Beneficial Effects of Scutellaria baicalensis on Penile Erection in Streptozotocin-Induced Diabetic Rats. Am J Chin Med 2016;44:305-320.

5 Pajovic B, Dimitrovski A, Fatic N, Malidzan M, Vukovic M: Vacuum erection device in treatment of organic erectile dysfunction and penile vascular differences between patients with DM type I and DM type II. Aging Male 2017;20:49-53.

6 Shamloul R, Ghanem H: Erectile dysfunction. Lancet 2013;381:153-165.

7 Yin GN, Park SH, Song KM, Limanjaya A, Ghatak K, Minh NN, Ock J, Ryu JK, Suh JK: Establishment of in vitro model of erectile dysfunction for the study of high-glucose-induced angiopathy and neuropathy. Andrology 2017;5:327-335.

-8 Wen J, Wang B, Du C, Xu G, Zhang Z, Li Y, Zhang N: A2B Adenosine Receptor Agonist Improves Erectile Function in Diabetic Rats. Tohoku J Exp Med 2015;237:141-148.

-9 Horiuchi M, Iwanami J, Mogi M: Regulation of angiotensin II receptors beyond the classical pathway. Clin Sci (Lond) 2012;123:193-203.

10 Benigni A, Cassis P, Remuzzi G: Angiotensin II revisited: new roles in inflammation, immunology and aging. EMBO Mol Med 2010;2:247-257.

11 Ramkumar N, Stuart D, Calquin M, Wang S, Niimura F, Matsusaka T, Kohan DE: Possible role for nephronderived angiotensinogen in angiotensin-II dependent hypertension. Physiol Rep 2016;4:pii: e12675.

12 Ferrario CM, VonCannon J, Jiao Y, Ahmad S, Bader M, Dell'Italia LJ, Groban L, Varagic J: Cardiac angiotensin-(1-12) expression and systemic hypertension in rats expressing the human angiotensinogen gene. Am J Physiol Heart Circ Physiol 2016;310:H995-1002.

13 Li R, Cui K, Wang T, Wang SG, Yang WM, Liu JH, Rao K: [Mechanism of RhoA/Rho-kinase signal pathwayinduced erectile dysfunction in hyperlipidemic rats]. Zhonghua Yi Xue Za Zhi 2016;96:2094-2097.

-14 Zhang Z, Xue HL, Liu Y, Wang WJ: Yi-Qi-Zeng-Min-Tang, a Chinese medicine, ameliorates insulin resistance in type 2 diabetic rats. World J Gastroenterol 2011;17:987-995.

-15 Putzbach W, Gao QQ Patel M, van Dongen S, Haluck-Kangas A, Sarshad AA, Bartom ET, Kim KA, Scholtens DM, Hafner M, Zhao JC, Murmann AE, Peter ME: Many si/shRNAs can kill cancer cells by targeting multiple survival genes through an off-target mechanism. Elife 2017;6:pii: e29702.

16 Fraga-Silva RA, Montecucco F, Mach F, Santos RA, Stergiopulos N: Pathophysiological role of the reninangiotensin system on erectile dysfunction. Eur J Clin Invest 2013;43:978-985.

17 Labazi H, Wynne BM, Tostes R, Webb RC: Metformin treatment improves erectile function in an angiotensin II model of erectile dysfunction. J Sex Med 2013;10:2154-2164.

18 Santos RA, Ferreira AJ, Simoes ESAC: Recent advances in the angiotensin-converting enzyme 2-angiotensin(1-7)-Mas axis. Exp Physiol 2008;93:519-527.

19 Iwai M, Horiuchi M: Devil and angel in the renin-angiotensin system: ACE-angiotensin II-AT1 receptor axis vs. ACE2-angiotensin-(1-7)-Mas receptor axis. Hypertens Res 2009;32:533-536.

-20 Ferrario CM: ACE2: more of Ang-(1-7) or less Ang II? Curr Opin Nephrol Hypertens 2011;20:1-6. 
21 Nunes KP, Rigsby CS, Webb RC: RhoA/Rho-kinase and vascular diseases: what is the link? Cell Mol Life Sci 2010;67:3823-3836.

22 Amano M, Nakayama M, Kaibuchi K: Rho-kinase/ROCK: A key regulator of the cytoskeleton and cell polarity. Cytoskeleton (Hoboken) 2010;67:545-554.

-23 Zhang WC, Peng YJ, Zhang GS, He WQ Qiao YN, Dong YY, Gao YQ, Chen C, Zhang CH, Li W, Shen HH, Ning W, Kamm KE, Stull JT, Gao X, Zhu MS: Myosin light chain kinase is necessary for tonic airway smooth muscle contraction. J Biol Chem 2010;285:5522-5531.

24 Rattan S, Phillips BR, Maxwell PJt: RhoA/Rho-kinase: pathophysiologic and therapeutic implications in gastrointestinal smooth muscle tone and relaxation. Gastroenterology 2010;138:13-18.e11-13.

25 Li WJ, Xu M, Gu M, Zheng DC, Guo J, Cai Z, Wang Z: Losartan Preserves Erectile Function by Suppression of Apoptosis and Fibrosis of Corpus Cavernosum and Corporal Veno-Occlusive Dysfunction in Diabetic Rats. Cell Physiol Biochem 2017;42:333-345.

26 Pan L, Ma J, Pan F, Zhao D, Gao J: Long Non-Coding RNA Expression Profiling in Aging Rats with Erectile Dysfunction. Cell Physiol Biochem 2015;37:1513-1526. 